\title{
Spin polarization of platinum (111) induced by the proximity to cobalt nanostripes
}

\author{
Focko Meier, ${ }^{1}$ Samir Lounis, ${ }^{2}$ Jens Wiebe, ${ }^{1,{ }^{*}}$ Lihui Zhou, ${ }^{1}$ Swantje Heers, ${ }^{3}$ Phivos Mavropoulos, ${ }^{3}$ Peter H. Dederichs, ${ }^{3}$ \\ Stefan Blügel, ${ }^{3}$ and Roland Wiesendanger ${ }^{1}$ \\ ${ }^{1}$ Institute of Applied Physics, Hamburg University, Jungiusstrasse 11, D-20355 Hamburg, Germany \\ ${ }^{2}$ Department of Physics and Astronomy, University of California Irvine, California 92697, USA \\ ${ }^{3}$ Institut für Festkörperforschung \& Institute for Advanced Simulation, Forschungszentrum Jülich \& JARA, D-52425 Jülich, Germany
}

(Received 7 October 2010; published 7 February 2011)

\begin{abstract}
We measured a spin polarization above a $\operatorname{Pt}(111)$ surface in the vicinity of a Co nanostripe by spin-polarized scanning tunneling spectroscopy. The spin polarization exponentially decays away from the Pt-Co interface and is detectable at distances larger than $1 \mathrm{~nm}$. By performing self-consistent $a b$ initio calculations of the electronic structure for a related model system we reveal the interplay between the induced magnetic moments within the Pt surface and the spin-resolved electronic density of states above the surface.
\end{abstract}

DOI: 10.1103/PhysRevB.83.075407

PACS number(s): 73.20.-r, 75.70.Cn, 75.75.-c

\section{INTRODUCTION}

The remarkable properties of magnetic nanostructures grown on nonmagnetic metal substrates rely significantly on the electronic coupling between the atoms within the nanostructure and the substrate atoms underneath. ${ }^{1}$ This electronic coupling determines, e.g., the strength and direction of the magnetic anisotropy as well as the total magnetic moment. ${ }^{2}$ Additionally the substrate electrons govern the collective behavior of ensembles of magnetic nanostructures, e.g., by providing ferromagnetic order due to indirect exchange interaction between separated magnetic nanostructures. ${ }^{3}$ This interaction, also known as the Ruderman-Kittel-KasuyaYosida (RKKY) interaction, has been found in diluted magnetic systems, where magnetic $3 \mathrm{~d}$ impurity atoms are dissolved in nonmagnetic host metals. ${ }^{4-6}$ In these samples, the localized magnetic moment of an impurity atom is screened by a spatially oscillating long-range spin polarization of the host conduction electrons. ${ }^{7}$ Therefore the distance between impurity atoms determines the sign and the strength of the interaction. The same coupling has recently been observed directly for atoms on surfaces. ${ }^{8,9}$ A second important effect takes place for magnetic impurity atoms in host metals which nearly fulfill the Stoner criterion, such as Pt and Pd; i.e., they are nearly ferromagnetic and are therefore characterized by a high susceptibility. In these so called giant-moment dilute alloys the impurities induce relatively strong magnetic moments in the neighboring host atoms which form a spin-polarized cluster. ${ }^{10}$ Since this effect can cause an additional exchange interaction between magnetic atoms in nanostructures it is important to obtain knowledge about the size of the polarization cloud and the decay of the induced magnetization with increasing distance from the magnetic atom. ${ }^{11,12}$

Both mechanisms are considered to be important for multilayer systems, ${ }^{13}$ like Co-Pt, which consist of sequences of ferromagnetic Co layers separated by nonmagnetic $\mathrm{Pt}$ spacer layers. ${ }^{14,15}$ The magnetic interlayer coupling between the ferromagnetic layers often shows deviations from a pure RKKY behavior, indicating that other mechanisms contribute to the total magnetic interaction. One contribution originates from magnetoelastic interactions due to interface roughness between the magnetic and nonmagnetic layers, ${ }^{16,17}$ while with decreasing temperatures the induced magnetic moments of
Pt become relevant for the magnetic coupling. ${ }^{18}$ In order to qualify specific contributions to the overall interaction a profound knowledge of the local configuration of the interface is required.

In this work we present a combined experimental and theoretical study on the spin polarization of $\mathrm{Pt}$ in the vicinity of Co nanostripes on a $\mathrm{Pt}$ (111) surface. We use spin-resolved scanning tunneling spectroscopy ${ }^{19}$ and the Korringa-KohnRostoker Green function method (KKR) within the framework of density functional theory (DFT). ${ }^{20}$ Our experimental technique allows one to obtain extensive knowledge concerning the topographic, electronic, and magnetic properties of the sample. We show that the measured Pt local electronic density of states (LDOS) near the Fermi energy in the vacuum exhibits an exponentially decaying spin polarization, indicating magnetic moments induced by the Co nanostripe. Interestingly this effect can be observed for lateral distances from the Co nanostripe larger than four Pt lattice spacings where the RKKY interaction provides already an antiferromagnetic coupling as shown in a previous study. ${ }^{8}$ The calculated induced magnetic moments in the Pt surface close to embedded Co atoms show a distance-dependent oscillation between ferromagnetic and antiferromagnetic alignment, while the vacuum spin polarization at particular energies experiences an exponential decay in the lateral direction.

\section{EXPERIMENTAL SETUP}

All experiments were performed in an ultrahigh-vacuum system containing a home-built $300-\mathrm{mK}$ scanning tunneling microscope (STM) operating at a magnetic field $B$ up to 12 T perpendicular to the sample surface. ${ }^{21}$ In this work we used Cr-covered W tips, which are sensitive to the out-of-plane direction of the nanostripe magnetization $\vec{M}_{\mathrm{Co}} \cdot{ }^{22,23} \mathrm{In}$ order to retain a strong spin polarization the tips were dipped into Co nanostripes. ${ }^{8,24}$ This procedure can result in a Co cluster attached to the tip apex, which affects the magnetic $B$ field required to switch the tip magnetization $\vec{M}_{\text {tip }}$. Further details on the sample and tip preparation are given in Refs. 8 and 24. Co was evaporated at two different temperatures on a clean $\operatorname{Pt}(111)$ crystal. First, a tenth of an atomic layer (AL) was deposited at room temperature leading to Co nanostripes 
attaching to the $\operatorname{Pt}(111)$ step edges. At a temperature below $25 \mathrm{~K}$ a much smaller amount was evaporated, which resulted in a tiny number of single Co adatoms randomly distributed on the surface.

\section{EXPERIMENTAL RESULTS}

Figure 1(a) shows a Co nanostripe attached to a Pt step edge between two $\mathrm{Pt}$ terraces and individual Co adatoms. The one AL high Co nanostripe can be easily identified by a dense network of dislocation lines originating from the lattice mismatch between the $\mathrm{Co}$ and the $\mathrm{Pt}^{24-26} \mathrm{~A}$ careful analysis of line sections as shown in Fig. 1(b) reveals that the Co stripe appears $20 \mathrm{pm}$ higher than the $\mathrm{Pt}$ layer to the right. Information regarding the spin-resolved LDOS in the vacuum above the Co nanostripe as well as

(a)

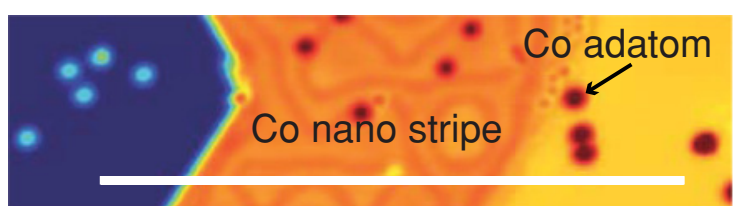

(b)

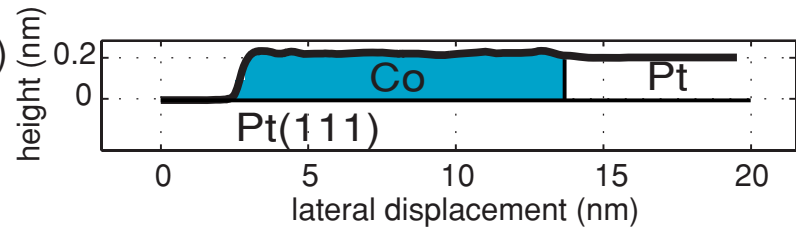

(c)

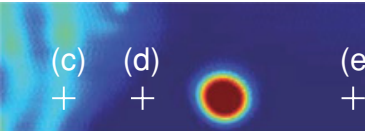

$$
B !+0.2 T \quad B=-0.2 T
$$

(d)

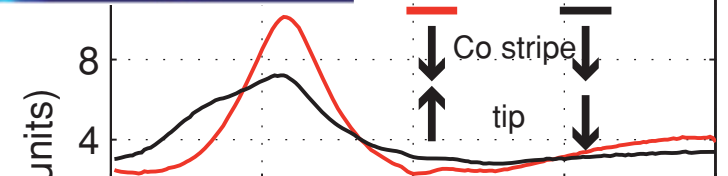

(e)

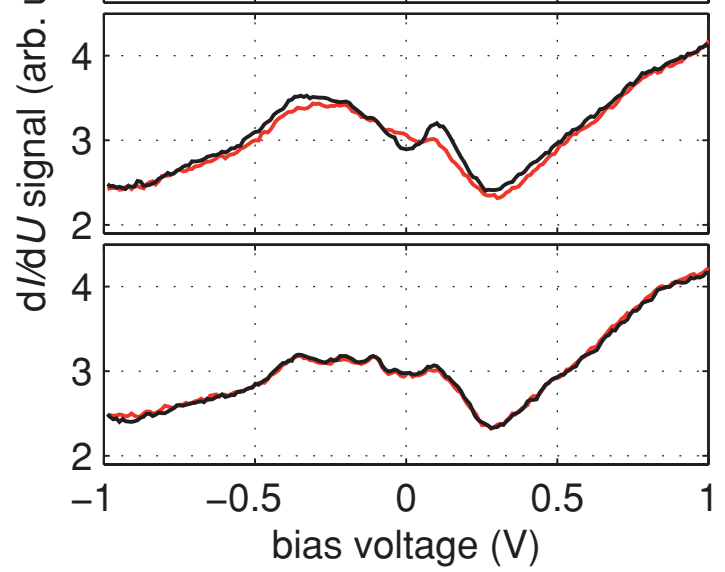

FIG. 1. (Color online) (a) STM topograph of two Pt(111) terraces with individual Co adatoms and a Co nanostripe attached to a Pt step edge. (b) Line section along the line marked in panel (a). (c)-(e) $d I / d U$ spectra taken at positions given in the inset, which displays the interface between the Co nanostripe (left) and the Pt terrace (right). The relative orientations of tip and Co nanostripe magnetization, $\vec{M}_{\text {tip }}$ and $\vec{M}_{\mathrm{Co}}$, are indicated by arrows. (Tunneling parameters: $U_{\text {stab }}=$ $1.0 \mathrm{~V}$, lock-in modulation voltage $U_{\text {mod }}=10 \mathrm{mV}$, stabilization current $I_{\text {stab }}=1.0 \mathrm{nA}, T=0.3 \mathrm{~K}$.) above the $\mathrm{Pt}$ surface is obtained by measuring the differential conductance $d I / d U$ as a function of location $\mathbf{r}$, the applied bias voltage $U_{\text {stab }}$, and the relative orientation between the tip magnetization $\vec{M}_{\text {tip }}$ and the nanostripe magnetization $\vec{M}_{\mathrm{Co}} \cdot{ }^{27}$ From previous measurements on nanostripes it is known that $\vec{M}_{\mathrm{Co}}$ is oriented out-of-plane. ${ }^{24}$ The relative orientations of $\vec{M}_{\text {tip }}$ and $\vec{M}_{\text {Co }}$ as well as their switching fields are determined unambiguously from magnetization curve measurements as described in Ref. 8.

Figures 1(c)-1(e) show the resulting $d I / d U(\mathbf{r}, U)$ spectra taken on locations, indicated in the inset, on the Co nanostripe and on the $\operatorname{Pt}(111)$ close and far from the nanostripe. For this particular tip, $\vec{M}_{\text {tip }}$ is switched up or down by $B$ fields of +0.2 and -0.2 T while $\vec{M}_{\text {Co }}$ is constant. This allows one to measure the $d I / d U$ signal for parallel and antiparallel alignment of $\vec{M}_{\text {tip }}$ and $\vec{M}_{\mathrm{Co}}$. On the Co nanostripe [cf. Fig. 1(c)] the spin-resolved $d I / d U$ spectra show a dominant peak located at $-0.4 \mathrm{eV}$ below $E_{\mathrm{F}}$ which originates from a $d$-like Co surface resonance of minority-spin character. ${ }^{24}$ The intensity of this state changes considerably for parallel and antiparallel alignment of $\vec{M}_{\text {tip }}$ and $\vec{M}_{\mathrm{Co}}$. In contrast to that, the spectra on the bare Pt far from the nanostripe in Fig. 1(e) do not show the electronic signature of the $d$-like surface resonance but the onset of the unoccupied surface state at $e U=0.3 \mathrm{eV}$ is visible. ${ }^{28}$ Furthermore, no dependency on $\vec{M}_{\text {tip }}$ is found as expected for a nonmagnetic material. Figure 1(d) shows spectra which have been taken on Pt but at a distance of only $1 \mathrm{~nm}$ with respect to the Co nanostripe. The spectra show the typical signature of a bare $\mathrm{Pt}(111)$ surface far from the Co stripe [cf. Fig. 1(e)]. However, a dependency on the relative orientation of $\vec{M}_{\text {tip }}$ and $\vec{M}_{\mathrm{Co}}$ is now observed in an energy range from -0.5 to $+0.5 \mathrm{eV}$ around $E_{\mathrm{F}}$. This effect is reproducible as shown by repeatedly recording spectra for parallel and antiparallel orientation and is larger than the error in the $d I / d U$ curves shown, which are averaged from four single spectra. Neither from our topographic nor spectroscopic data do we have any indications of Co incorporation into the Pt surface or subsurface layers within the probed area. ${ }^{29,30}$ This experimental result already proves a spin polarization of the clean $\mathrm{Pt}(111)$ at a distance of more than three lattice spacings to the Co nanostripe.

In order to obtain information about this induced spin polarization we probed the spatially resolved $d I / d U$ signal $(d I / d U$ map) in a boundary area shown in Fig. 2(a). For this area $d I / d U$ maps have been recorded at $U_{\text {stab }}=+0.3 \mathrm{~V}$ in a complete $B$-field loop starting from $-0.8 \mathrm{~T}$, then going to $+1.0 \mathrm{~T}$ and back to $-2.0 \mathrm{~T}$ (after first applying $-2.5 \mathrm{~T}$ in order to magnetize $\vec{M}_{\text {tip }}$ downward). Figures 2(b) and 2(c) show exemplary three-dimensional (3D) topographs colorized with the simultaneously measured $d I / d U$ maps obtained at $B=+0.6 \mathrm{~T}$ and $B=+1.0 \mathrm{~T}$, where the relative orientation of $\vec{M}_{\text {tip }}$ and $\vec{M}_{\mathrm{Co}}$ has changed due to a $B$-field-induced $\vec{M}_{\mathrm{Co}}$ reversal (for this particular tip, $\vec{M}_{\text {tip }}$ is not switched up to $|B|=2 \mathrm{~T})$. The $d I / d U$ signal above the Pt terrace appears to be the same in both figures. However, a difference in $d I / d U$ intensity above Pt close to the stripe is observed as shown by the white rectangle.

From the sequence of $B$-field-dependent $d I / d U$ maps local magnetization curves are obtained by plotting the $d I / d U$ 


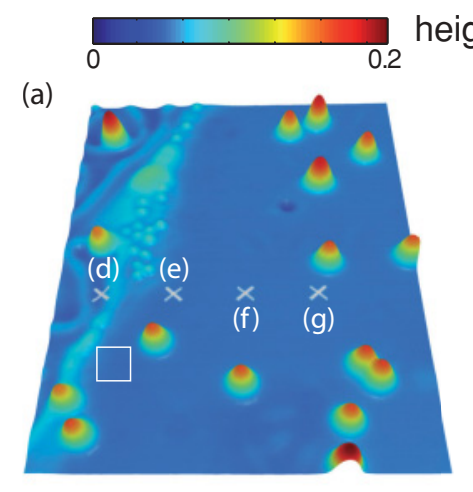

(d)

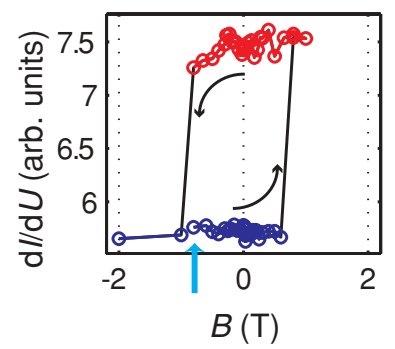

(e)

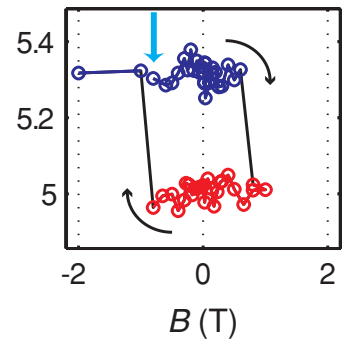

4.5

(b)

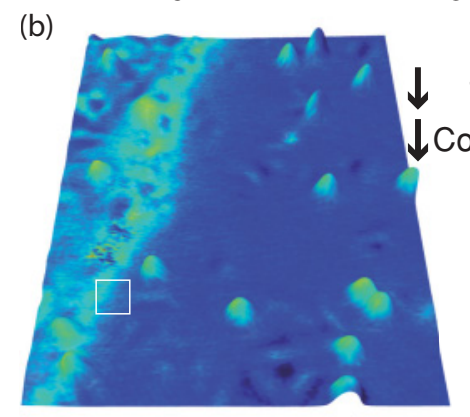

(f)

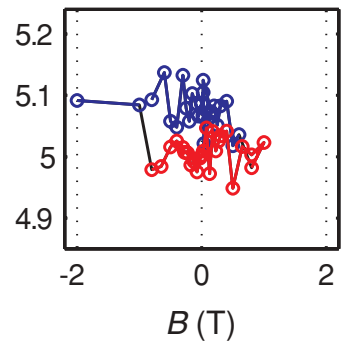

$d / / d U$ signal (arb. units)

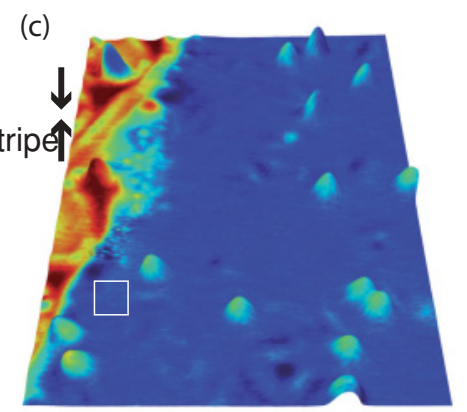

(g)

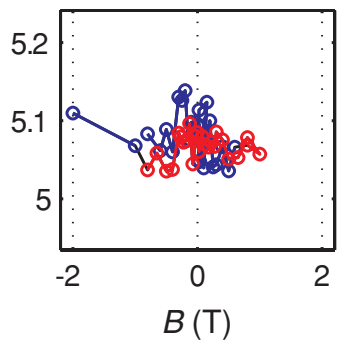

FIG. 2. (Color online) (a) STM topograph in 3D view (size $11.6 \times 15.6 \mathrm{~nm}^{2}$ ). (b) and (c) STM topograph in 3D view colorized with the simultaneously recorded spin-resolved $d I / d U$ map obtained at $B=+0.6 \mathrm{~T}$ and $B=+1.0 \mathrm{~T}$, respectively. The white rectangles mark exactly the same area in panels (a)-(c). Relative orientation of $\vec{M}_{\text {tip }}$ and $\vec{M}_{\text {Co }}$ is indicated by arrows. (Tunneling parameters: $U_{\text {stab }}=0.3 \mathrm{~V}$, $U_{\text {mod }}=20 \mathrm{mV}, I_{\text {stab }}=0.8 \mathrm{nA}$.) (d)-(g) Magnetization curves taken at positions marked by crosses in Fig. 1(a). Positions are separated by $2.3 \mathrm{~nm}$. Arrows in panels (d) and (e) mark the start and direction of rotation of the $B$ field loop. Blue and red colors indicate $d I / d U$ values representing parallel and antiparallel orientation of $\vec{M}_{\text {tip }}$ and $\vec{M}_{\text {Co }}$ for each hysteresis.

signal at one image point as a function of $B$. Figures 2(d)$2(\mathrm{~g})$ show local magnetization curves taken at positions as marked in Fig. 2(a). The magnetization curve of the Co stripe in Fig. 2(d) shows two magnetic states and a squarelike hysteresis indicating its ferromagnetic state and a coercivity of $B_{c}=0.7 \pm 0.05 \mathrm{~T}$. Strikingly, the magnetization curves measured on the Pt in the vicinity of the Co nanostripe show that there is an explicit link between the magnetic state of the Co stripe and the spin polarization measured on the Pt. Similar magnetization curves have been recorded for each point of the area shown in Fig. 2(a). From these magnetization curves the so-called spin asymmetry $A_{\text {spin }}$ is calculated by

$$
A_{\text {spin }}=\frac{d I / d U_{\uparrow \uparrow}-d I / d U_{\uparrow \downarrow}}{d I / d U_{\uparrow \uparrow}+d I / d U_{\uparrow \downarrow}}
$$

which characterizes the squarelike magnetization curves and is a measure for the spin-polarization at $e U$ in the vacuum. ${ }^{23}$ $d I / d U_{\uparrow \uparrow}$ and $d I / d U_{\uparrow \downarrow}$ denote the averaged values from all blue and red data points in the magnetization curves [Figs. 2(d)-2(g)], i.e., for parallel and antiparallel alignment of $\vec{M}_{\text {tip }}$ and $\vec{M}_{\text {Co }}$ in each curve. An asymmetry value is obtained for each image point. This results in an asymmetry map shown in Fig. 3(a). The Co stripe shows a strong negative $A_{\text {spin }}$ while on the Pt terrace far from the stripe $A_{\text {spin }}$ is zero. Above the $\mathrm{Pt}$ close to the Co stripe an area with positive $A_{\text {spin }}$ is visible which fades out for an increasing distance from the nanostripe. The decay is further analyzed in Fig. 3(b), which shows $A_{\text {spin }}$ values below the section line in Fig. 3(a) as a function of the distance $d$ from the Co nanostripe. In order to quantify the decay behavior the graph in Fig. 3(b) has been fitted to the simple exponential function

$$
f=C e^{-x / \lambda},
$$

where $C$ and $\lambda$ denote the amplitude and the decay length, respectively. Even though the exact value of $\lambda$ depends on the specific line section, values in the range from $\lambda=0.9 \mathrm{~nm}$ to $\lambda=1.2 \mathrm{~nm}$ are obtained corresponding to more than three next-nearest-neighbor distances within the Pt lattice. We observe the same quantitative behavior in $A_{\text {spin }}$ calculated from $d I / d U(\mathbf{r})$ maps recorded at $U_{\text {stab }}=-0.1 \mathrm{~V}$ [see inset in Fig. 3(b)]. Together with the dependency on the spin-resolved $d I / d U$ curves measured close to the Co stripe [see Fig. 1(d)], we conclude that the observed spin polarization above $\mathrm{Pt}$ is present in a large energy window around the Fermi energy. This result suggests that the measured spin polarization is due to an exponentially decaying magnetic moment $\vec{M}_{\mathrm{Pt}}$ induced by the vicinity to the Co nanostripe.

Figure 3(b) also includes the experimentally obtained indirect exchange energies, $J$, between the Co nanostripe and single Co adatoms on Pt(111) as published in Ref. 8. A positive $J$ corresponds to a ferromagnetic coupling while a negative value corresponds to an antiferromagnetic coupling. A damped oscillatory exchange interaction is present in the same range where the exponentially decaying $\mathrm{Pt}$ vacuum spin polarization is measured. It is shown in Ref. 8 that the exchange interaction can be described by RKKY-like 


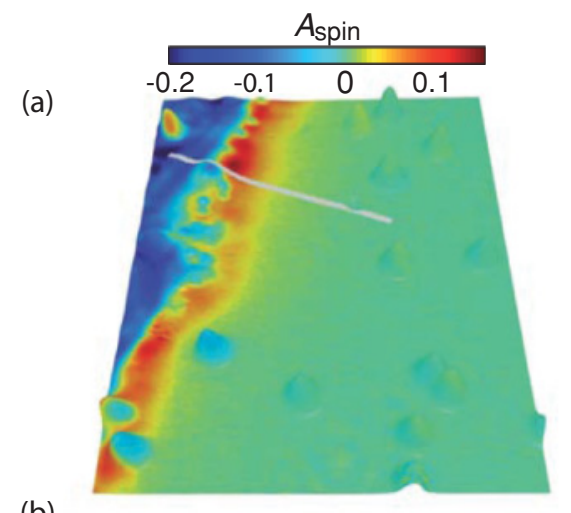

(b)

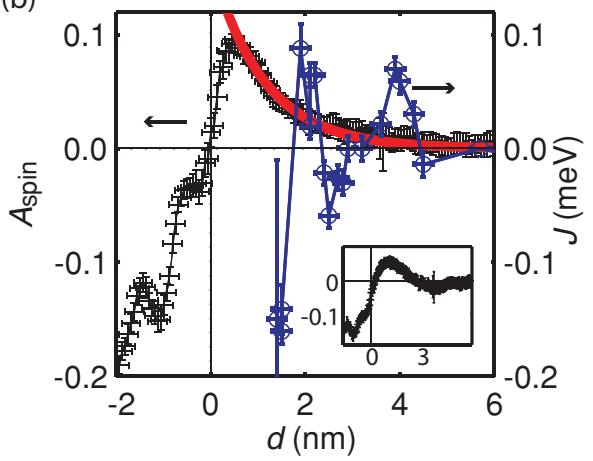

FIG. 3. (Color online) (a) STM topograph in 3D view colorized with the calculated asymmetry map obtained from local magnetization curves $\left(U_{\text {stab }}=0.3 \mathrm{~V}\right)$. (b) Crosses: Asymmetry values below line section indicated in panel (a). Open circles: Magnetic exchange energy $J$ for the coupling between Co nanostripe and individual Co atoms taken from Ref. 8. "0" indicates the border between Co nanostripe and Pt layer. The red (thick) line shows an exponential fit according to Eq. (2). Inset: Asymmetry values as in panel (a) for $U_{\text {stab }}=-0.1 \mathrm{~V}$.

exchange and follows in good agreement a one-dimensional range function. In the case of a strong contribution of a ferromagnetic Pt polarization one would expect a dominance of ferromagnetic coupling for the overall magnetic exchange interaction. Such an effect would become visible by a shift of the RKKY curve toward positive exchange energies, which is not observed. These observations raise the following question: how exactly is the measured Pt spin polarization linked to the induced magnetization within the Pt surface?

\section{THEORETICAL METHOD}

In order to obtain deeper insight into the relation between the measured spin polarization in the vacuum and the induced magnetization we performed calculations on three different arrangements of $\mathrm{Co}$ on or in a $\mathrm{Pt}(111)$ surface layer as shown in Fig. 4(a). First, we considered a single Co atom deposited on (adatom) and embedded in (inatom) the first layer of $\mathrm{Pt}(111)$. These two arrangements differ mainly in the number of next-neighbor Pt atoms, which is tripled for the inatom with respect to the adatom case. Therefore a comparison of these two cases provides us with important information concerning the hybridization of the Co electronic states with those of the $\mathrm{Pt}$ surface leading to the magnetization of the surrounding $\mathrm{Pt}$ atoms.
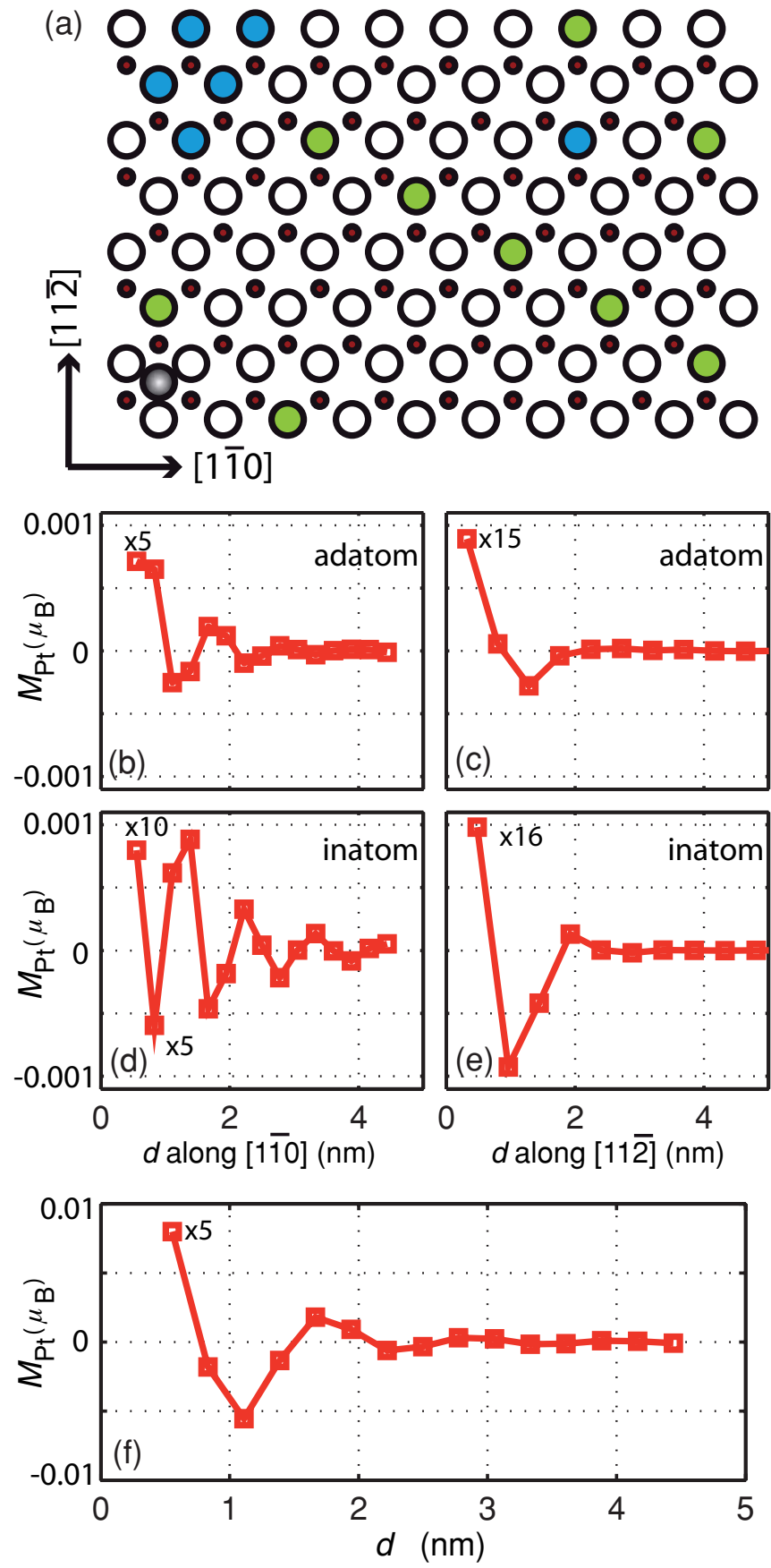

FIG. 4. (Color online) (a) Sketch of the three considered sample setups for calculations. Large open and small red circles represent $\mathrm{Pt}(111)$ surface and subsurface atoms, respectively. Solid blue circles indicate the locations of the embedded Co atom chain and the Co inatom. The gray circle marks the position of the Co adatom. Solid green circles indicate the closest considered atoms for the calculation of the induced moments in each specific direction. (b)-(e) Induced magnetic moments in $\mathrm{Pt}$ atoms $M_{\mathrm{Pt}}$ for two indicated directions as a function of distance $d$ from a Co adatom and a Co inatom. (f) $M_{\mathrm{Pt}}$ as a function of distance $d$ from the embedded Co chain for experimentally relevant direction. Some values in panels (b)-(f) have been scaled down by the indicated factors in order to fit into the figure.

In order to model the experimental setup as closely as possible we constructed a chain of five Co atoms embedded in the surface of $\mathrm{Pt}(111)$ [see Fig. 4(a)]. This model arrangement 
reflects the experimental fact that the Pt surface atoms which show a vacuum spin polarization are located in the same layer as the Co atoms which form the nanostripe. The chain is oriented along a direction perpendicular to the direction probed experimentally concerning the spin polarization [see Fig. 3(a)]. The exact experimental setup is of course difficult to achieve since a nonregular step edge of platinum interfacing a cobalt stripe is impossible to reproduce with methods based on DFT at the actual stage.

The method of investigation is the KKR method ${ }^{20}$ within the framework of DFT. The KKR method is based on multiplescattering theory. For non-overlapping potentials the following angular momentum representation of the Green's function $G\left(\mathbf{r}+\mathbf{R}_{n}, \mathbf{r}^{\prime}+\mathbf{R}_{n^{\prime}} ; E\right)$ can be derived:

$$
\begin{aligned}
G\left(\mathbf{r}+\mathbf{R}_{n}, \mathbf{r}^{\prime}+\mathbf{R}_{n^{\prime}} ; E\right)= & -i \sqrt{E} \sum_{L} R_{L}^{n}\left(\mathbf{r}_{<} ; E\right) H_{L}^{n}\left(\mathbf{r}_{>} ; E\right) \delta_{n n^{\prime}} \\
& +\sum_{L L^{\prime}} R_{L}^{n}(\mathbf{r} ; E) G_{L L^{\prime}}^{n n^{\prime}}(E) R_{L^{\prime}}^{n^{\prime}}\left(\mathbf{r}^{\prime} ; E\right) .
\end{aligned}
$$

Here $\mathbf{R}_{n}$ and $\mathbf{R}_{n^{\prime}}$ refer to the atomic positions and $E$ is the energy. $\mathbf{r}_{<}$and $\mathbf{r}_{>}$denote the shorter and longer of the vectors $\mathbf{r}$ and $\mathbf{r}^{\prime}$ which define the position in the Wigner-Seitz cell centered around $\mathbf{R}_{n}$ or $\mathbf{R}_{n}^{\prime} . R_{L}^{n}(\mathbf{r} ; E)$ and $H_{L}^{n}(\mathbf{r} ; E)$ are the regular and irregular solutions of the Schrödinger equation.

The structural Green functions $G_{L L^{\prime}}^{n n^{\prime}}(E)$ are then obtained by solving the Dyson equation for each spin direction:

$$
\begin{aligned}
G_{L L^{\prime}}^{n n^{\prime}}(E)= & g_{L L^{\prime}}^{n n^{\prime}}(E) \\
& +\sum_{n^{\prime \prime}, L^{\prime \prime} L^{\prime \prime \prime}} g_{L L^{\prime \prime}}^{n n^{\prime \prime}}(E) \Delta t_{L^{\prime \prime} L^{\prime \prime \prime}}^{n^{\prime \prime}}(E) G_{L^{\prime \prime \prime} L^{\prime}}^{n^{\prime \prime} n^{\prime}}(E) .
\end{aligned}
$$

The summation in (4) is over all lattice sites $n^{\prime \prime}$ and angular momenta $L^{\prime \prime}$ and $L^{\prime \prime \prime}$, for which the perturbation $\Delta t_{L^{\prime \prime} L^{\prime \prime \prime}}^{n^{\prime \prime}}(E)$ between the $t$ matrices of the real and the reference system is significant. $g_{L L^{\prime}}^{n n^{\prime}}$ is the structural Green function of the reference system, i.e., in our case the ideal $\operatorname{Pt}(111)$ surface.

The real-space solution of the Dyson equation requires a cluster of perturbed atomic potentials that include the potential of Co impurities and the first shell of neighboring cells. It is important to note that the vacuum region is filled with cellular (Voronoi) potentials. Since our aim is to explain the STM measured spectra, we use the Tersoff-Hamann theory ${ }^{31,32}$ and calculate the local density of states in the vacuum at 4.1 Å above the substrate. After obtaining a self-consistent Co potential with its neighboring shell, one additional calculation is performed including $\mathrm{Pt}$ atoms as well as their neighboring vacuum cells at $4.1 \AA$ above the substrate along a given direction.

\section{THEORETICAL RESULTS}

For an individual Co adatom and an individual Co inatom we calculated the induced magnetic moments $M_{\mathrm{Pt}}$ in the $\mathrm{Pt}$

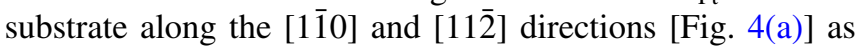
a function of the distance $d$ from the impurity shown in Figs. 4(b)-4(e). Concerning the [110] direction we find for both arrangements a long-range oscillation with a wavelength of about $1 \mathrm{~nm}$ for the adatom [see Fig. 4(b)] and a slightly smaller one for the inatom [see Fig. 4(d)]. The oscillation indicates that $M_{\mathrm{Pt}}$ is either ferromagnetically or antiferromagnetically aligned with the Co impurity dependent on the distance. However, the total integrated net moment of the $\mathrm{Pt}$ atoms is positive. Along the [112] direction the oscillatory behavior is much weaker for both arrangements [Figs. 4(c) and 4(e)]. Here more Pt atoms are coupled ferromagnetically to the Co impurity. This directional dependence proves that the induced magnetization is anisotropic due to the nonspherical Fermi surface characterizing this system as found in the directionally dependent RKKY interactions between Co adatoms on a $\operatorname{Pt}(111)$ surface or in the induced anisotropic charge oscillations caused by Co impurities buried below $\mathrm{Cu}$ surfaces. ${ }^{9,33}$ A comparison of Fig. 4(b) with Fig. 4(d) or of Fig. 4(c) with Fig. 4(e) for the same distances shows that $M_{\mathrm{Pt}}$ is always larger for the embedded atom than for the adatom. This emphasizes a dependence of the coupling between the Co and Pt electronic states on the coordination. To favor the coupling to the impurity states, the electronic states controlling the studied long-range $M_{\mathrm{Pt}}$ must be localized at the surface. Constant energy contours at the Fermi energy $E_{\mathrm{F}}$ are plotted in Fig. 5(a) for the simulated Pt(111) surface with their relative localization at the surface layer. There is a finite number of contours due to the fact that the surface is simulated with a finite number of Pt layers. The shape of the contours is nontrivial, indicating the complexity of the problem. This type of calculation indicates the presence of several resonant states. To measure the degree of coupling between these states and those of the Co impurity, we decompose the Fermi surface in ten parts represented within the red-yellow triangle in Fig. 5(a). Each part includes both weakly and strongly surface-localized states. Afterward, we calculate the magnetization at $E_{\mathrm{F}}$ induced by every part. The structural Green function $g$ of $\operatorname{Pt}(111)$ needed in Eq. (4) is given as a Fourier transform or integral over the first Brillouin zone. This integration can be done for every region defined in Fig. 5(a), leading to values that can be plugged into Eqs. (3) and (4) to compute the contribution of every region to the magnetization of $\mathrm{Pt}$ at $E_{\mathrm{F}}$. For the inatom case parts 7,8 , and 10 are contributing most to the induced $M_{\mathrm{Pt}}$ [see Fig. 5(b)]. By summing up all parts, we approximately recover the total energy integrated magnetization [see Fig. 4(d)]. However, an exact recovery is not expected since with the decomposition scheme some scattering events cancel each other and other "backscattering" events are not taken into account properly. This theoretical experience demonstrates the nontrivial link between the induced long-range magnetization and the constant energy contours of the substrate, their degree of localization at the surface layers, and coupling strength with the impurities.

Figure 4(f) shows $M_{\mathrm{Pt}}$ for $\mathrm{Pt}$ atoms perpendicular to the embedded Co chain [Fig. 4(a)], as a function of distance $d$ from the chain, which is the setup closest to the experiment. In contrast to the experimentally observed exponential decay of the vacuum spin polarization, an oscillatory $M_{\mathrm{Pt}}$ is again calculated as in the cases before. However, $M_{\mathrm{Pt}}$ shows overall higher intensities which reflects contributions from all the $\mathrm{Co}$ atoms within the chain. In order to investigate the relation between $M_{\mathrm{Pt}}$ and the measured spin polarization we calculated the vacuum LDOS for majority and minority spin states above the $\mathrm{Pt}$ atoms along the direction perpendicular to the chain 


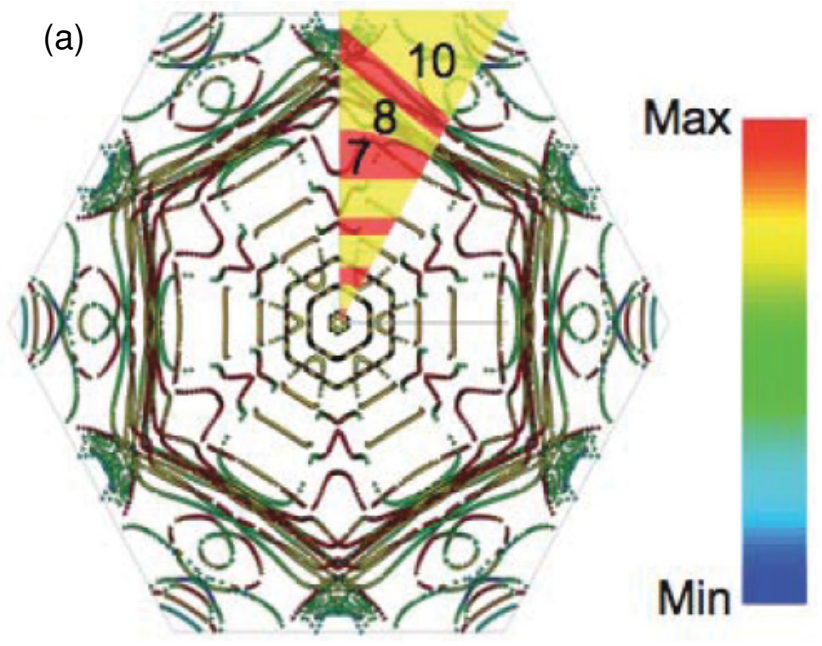

(b)

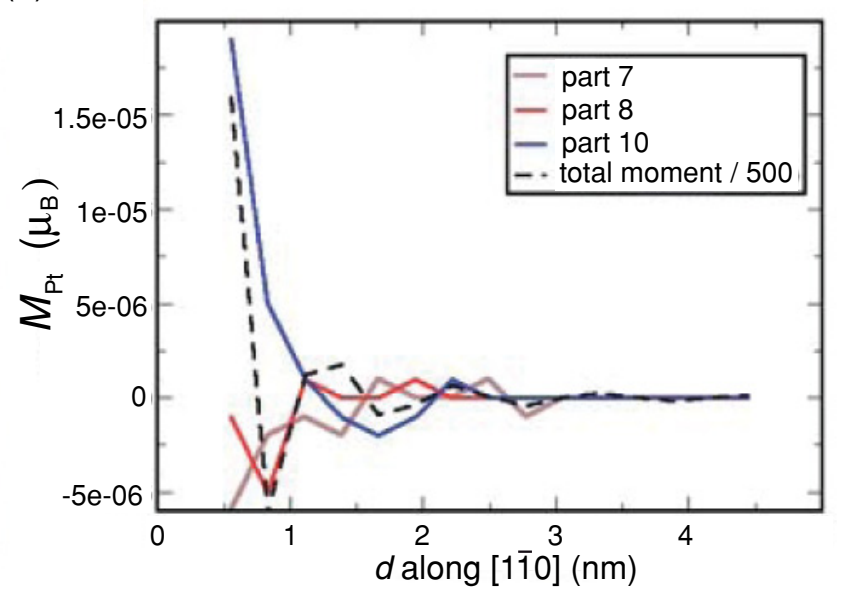

FIG. 5. (Color online) (a) Constant energy contours calculated at $E_{\mathrm{F}}$, where colors represent the degree of localization of the different electronic states at the surface layer of $\mathrm{Pt}(111)$. In addition, a triangle divided into ten regions is superimposed on the energy contours. Depending on the region considered, the induced $M_{\mathrm{Pt}}$ in the surrounding Pt surface atoms changes. As an example, we plot in panel (b) $M_{\mathrm{Pt}}$ along the $[1 \overline{1} 0]$ direction for the inatom case induced by the most contributing constant energy contours: regions 7,8 , and 10 .

at a vertical distance of $4.1 \AA$. This corresponds to two interlayer distances from the surface and is the range of the experimental $z$ height of the tip. Figures 6(a)-6(d) show the results for the first, second, third, and fifth Pt atom located
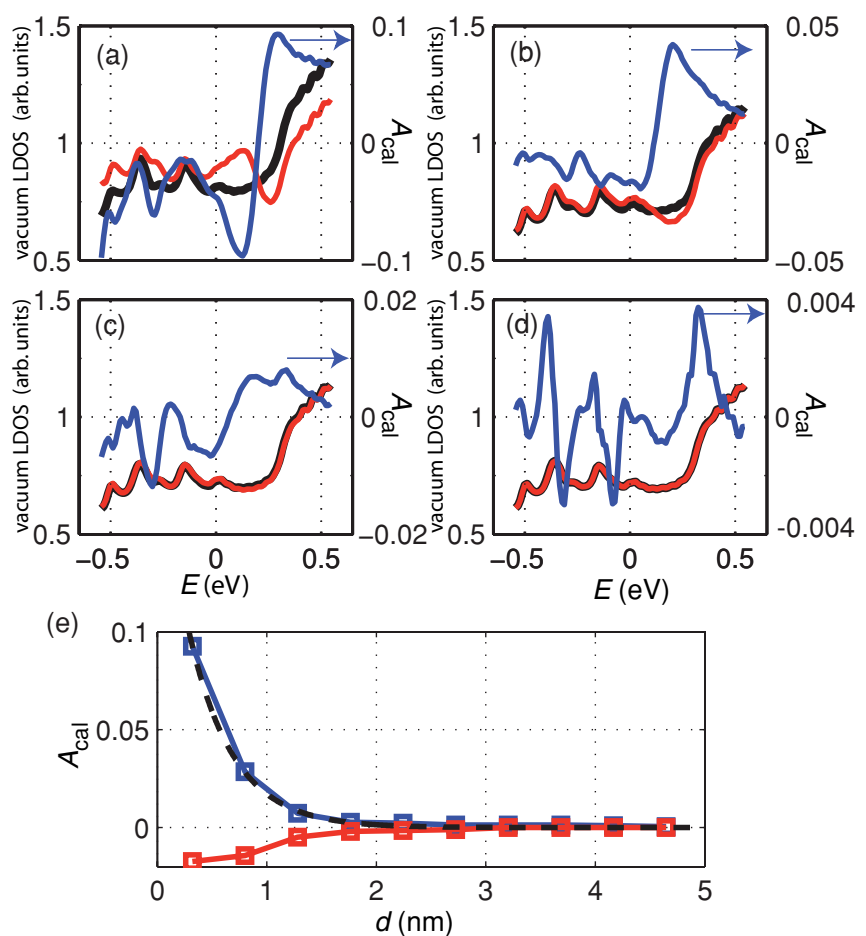

FIG. 6. (Color online) (a)-(d) Calculated vacuum LDOS $4.1 \AA$ above the first, second, third, and fifth Pt atom close to the embedded Co chain for the majority spin state (black) and the minority spin state (red) as well as the corresponding asymmetry $A_{\text {cal }}(E)$ (blue). (e) $A_{\text {cal }}$ plotted as a function of distance along the direction perpendicular to the chain at $+0.3 \mathrm{eV}$ (blue) and $-0.1 \mathrm{eV}$ (red). The dashed line shows an exponential fit according to Eq. (2). in the relevant direction. They reveal an intensity increase starting at about $+0.3 \mathrm{eV}$ which is due to the Pt surface state. ${ }^{28}$ The $\mathrm{Pt}$ atom closest to the chain experiences the strongest imbalance of majority and minority electrons. This is visualized by a corresponding calculated spin asymmetry $A_{\text {cal }}(E)$ given by

$$
A_{\mathrm{cal}}(E)=\frac{\operatorname{LDOS}_{\text {maj }}(E)-\operatorname{LDOS}_{\text {min }}(E)}{\operatorname{LDOS}_{\text {maj }}(E)+\operatorname{LDOS}_{\text {min }}(E)},
$$

where $\operatorname{LDOS}_{\text {maj }}(E)$ and $\operatorname{LDOS}_{\text {min }}(E)$ denote the energydependent vacuum LDOS for majority and minority electrons. $A_{\text {cal }}(E)$ is plotted in Figs. 6(a)-6(d) for the Pt atoms as well. These curves reveal that neither the absolute value nor the sign of $A_{\text {cal }}(E)$ is conserved when scanning at different bias voltages around the Fermi energy. Additionally the absolute value of $A_{\text {cal }}(E)$ at given energies changes with increasing distance from the Co chain. Figure 6(e) shows the calculated $A_{\text {cal }}(E)$ for +0.3 and $-0.1 \mathrm{eV}$, which are experimentally relevant, for different distances from the chain. A comparison of these curves with the experimental data obtained at $+0.3 \mathrm{~V}$ shown in Fig. 3(b) reveals that $A_{\text {cal }}(+0.3 \mathrm{eV})$ follows the shape of the experimental curve; i.e., it is always positive and shows an exponentially decaying behavior. A fit as in Eq. (2) gives a value for the decay length $\lambda$ of about $4 \AA$ which is about half of the experimental value. $A_{\text {cal }}(-0.1 \mathrm{eV})$ shows a similar behavior but with reversed sign. This change of sign in comparison to the experiment Fig. 3(b) is most likely due to a change of the tip spin polarization which is known to occur for a bias voltage range below the Fermi energy. ${ }^{34}$

\section{DISCUSSION}

Recently several theoretical studies have concentrated on probing and describing magnetic properties of Co nanostructures on $\mathrm{Pt}(111)$ quantitatively and qualitatively. They treated $\mathrm{Co}$ in different configurations and environments, like $\mathrm{Co}$ 
overlayers on $\operatorname{Pt}(111)^{35}$, Co nano wires attached to $\operatorname{Pt}(111)$ step edges ${ }^{36,37}$ and isolated Co adatoms on bare $\operatorname{Pt}(111)$ surfaces. ${ }^{38,39}$ Even though these configurations have different coordination numbers of underlying $\mathrm{Pt}$ atoms per Co atom, they show consistently an induced spin moment $M_{\text {spin }}$ of the nearest-neighbor Pt atoms in the range from 0.1 to $0.3 \mu_{B}$, which is about 1 order of magnitude larger than the orbital moments $M_{\mathrm{orb}}$. Therefore the total induced magnetic moment $M_{\mathrm{Pt}}$ of $\mathrm{Pt}$ atoms is mainly determined by the spin moment $M_{\text {spin }}$.

Additionally it has been found in these calculations that $M_{\mathrm{Pt}}$ decreases very rapidly with the distance from the Co structures by about 1 order of magnitude for the second and third nearest neighbors as shown for the Co nano wires in Ref. 36. Here we probed experimentally and theoretically $M_{\mathrm{Pt}}$ for longer distances far from the Co impurities. We find that $M_{\mathrm{Pt}}$ is not constantly parallelly or antiparallelly aligned with the magnetic moment of the Co impurity. The sign as well as the strength of $M_{\mathrm{Pt}}$ is additionally highly influenced by the strong anisotropy of the Fermi surface of Pt. Both underline that for the probed arrangements of Co on and in the $\operatorname{Pt}(111)$ surface one cannot expect a constantly aligned polarization cloud as found for Co-Pt and Fe-Ir multilayers. ${ }^{18,40}$

The apparent contradiction of the measured monotonously decaying $A_{\text {spin }}$ in the vacuum and the calculated oscillating $M_{\mathrm{Pt}}$ can be explained by local changes of the electronic structure of the Pt atoms close to the embedded chain [see Figs. 6(a)-6(d)]. It is evident also that the hybridization between the Pt and the Co states changes with increasing the distance from the chain. Therefore also the spin-averaged LDOS changes laterally and can be obtained by calculating the arithmetic mean of the LDOS for both spin types in Figs. 6(a)-6(d). According to Ref. 27 the measured spin-resolved $d I / d U$ signal and the deduced spin asymmetry is a measure of the energy-dependent spin polarization of the sample. This quantity is only a measure for the magnetization, which is an integrated quantity of majority and minority states up to the Fermi energy, if the spin-averaged LDOS is laterally constant. Therefore the induced magnetization of the Pt cannot be deduced from the experimentally detected vacuum spin polarization in the Pt only.

\section{CONCLUSIONS}

In conclusion, we have performed spin-polarized scanning tunneling spectroscopy on $\mathrm{Pt}(111)$ in proximity to Co nanostripes at $0.3 \mathrm{~K}$. Given the locally measured spin polarization above $\mathrm{Pt}$, it is possible to retrieve the induced magnetic moments in a nonmagnetic material on a local scale, with the help of theory. The measured vacuum spin polarization decays exponentially as a function of the distance from the Co nanostripe with a decay length of about $1 \mathrm{~nm}$.

Self-consistent electronic-structure calculations of a Co chain embedded in the $\operatorname{Pt}(111)$ surface, of the neighboring $\mathrm{Pt}$ atoms, and of the vacuum LDOS above the Pt allow us to prove that the measured spin polarization is induced by an oscillating and highly anisotropic magnetization within the $\mathrm{Pt}$ surface in proximity to Co. By investigating the Fermi surface contours of $\operatorname{Pt}(111)$ and their degree of localization on the surface layer, we found several states with anisotropic shapes that can couple to the electronic states of Co impurities and thus contribute to the long-range induced magnetization.

\section{ACKNOWLEDGMENTS}

We acknowledge financial support from the SFB 668 and GrK 1286 of the DFG, from the ERC Advanced Grant FURORE, and from the Cluster of Excellence "Nanospintronics" funded by the Forschungs- und Wissenschaftsstiftung Hamburg. S.L. wishes to thank the Alexander von Humboldt Foundation for a Feodor Lynen Fellowship and thanks D. L. Mills for discussions and hospitality. The computations were performed on the supercomputer JUROPA at the Forschungszentrum Jülich.
*Corresponding author: jwiebe@physnet.uni-hamburg.de

${ }^{1}$ S. Blügel, M. Weinert, and P. H. Dederichs, Phys. Rev. Lett. 60, 1077 (1988).

${ }^{2}$ P. Gambardella, S. Rusponi, M. Veronese, S. Dhesi, C. Grazioli, A. Dallmeyer, I. Cabria, R. Zeller, P. Dederichs, and K. Kern et al., Science 300, 1130 (2003).

${ }^{3}$ J. P. Pierce, M. A. Torija, Z. Gai, J. Shi, T. C. Schulthess, G. A. Farnan, J. F. Wendelken, E. W. Plummer, and J. Shen, Phys. Rev. Lett. 92, 237201 (2004).

${ }^{4}$ M. A. Ruderman and C. Kittel, Phys. Rev. 96, 99 (1954).

${ }^{5}$ T. Kasuya, Prog. Theor. Phys. 16, 45 (1956).

${ }^{6}$ K. Yosida, Phys. Rev. 106, 893 (1957).

${ }^{7}$ L. D. Graham and D. S. Schreiber, Phys. Rev. Lett. 17, 650 (1966).

${ }^{8}$ F. Meier, L. Zhou, J. Wiebe, and R. Wiesendanger, Science 320, 82 (2008).

${ }^{9}$ L. Zhou, J. Wiebe, S. Lounis, E. Vedmedenko, F. Meier, S. Blügel,

P. H. Dederichs, and R. Wiesendanger, Nat. Phys. 6, 187 (2010).

${ }^{10}$ J. Crangle, Philos. Mag. 5, 335 (1960).

${ }^{11}$ R. Skomski, J. Zhang, V. Sessi, J. Honolka, K. Kern, and A. Enders, J. Appl. Phys. 103, 07D519 (2008).
${ }^{12}$ A. Oswald, R. Zeller, and P. H. Dederichs, Phys. Rev. Lett. 56, 1419 (1986).

${ }^{13}$ S. S. P. Parkin, N. More, and K. P. Roche, Phys. Rev. Lett. 64, 2304 (1990).

${ }^{14}$ P. Bruno and C. Chappert, Phys. Rev. B 46, 261 (1992).

${ }^{15}$ K. Le Dang, P. Veillet, C. Chappert, R. F. C. Farrow, R. F. Marks, D. Weller, and A. Cebollada, Phys. Rev. B 50, 200 (1994).

${ }^{16}$ J. Moritz, F. Garcia, J. C. Toussaint, B. Dieny, and J. P. Nozires, Europhys. Lett. 65, 123 (2004).

${ }^{17}$ X.-X. Li, J. Bao, L.-Y. Lu, X.-G. Xu, and Y. Jiang, Solid State Commun. 148, 209 (2008).

${ }^{18}$ J. W. Knepper and F. Y. Yang, Phys. Rev. B 71, 224403 (2005).

${ }^{19}$ R. Wiesendanger, Rev. Mod. Phys. 81, 1495 (2009).

${ }^{20}$ N. Papanikolaou, R. Zeller, and P. H. Dederichs, J. Phys. Condens. Matter 14, 2799 (2002).

${ }^{21}$ J. Wiebe, A. Wachowiak, F. Meier, D. Haude, T. Foster, M. Morgenstern, and R. Wiesendanger, Rev. Sci. Instrum. 75, 4871 (2004).

${ }^{22}$ A. Kubetzka, M. Bode, O. Pietzsch, and R. Wiesendanger, Phys. Rev. Lett. 88, 057201 (2002). 
${ }^{23}$ M. Bode, Rep. Prog. Phys. 66, 523 (2003).

${ }^{24}$ F. Meier, K. von Bergmann, P. Ferriani, J. Wiebe, M. Bode, K. Hashimoto, S. Heinze, and R. Wiesendanger, Phys. Rev. B 74, 195411 (2006).

${ }^{25}$ P. Grütter and U. T. Dürig, Phys. Rev. B 49, 2021 (1994).

${ }^{26}$ E. Lundgren, B. Stanka, M. Schmid, and P. Varga, Phys. Rev. B 62, 2843 (2000).

${ }^{27}$ D. Wortmann, S. Heinze, P. Kurz, G. Bihlmayer, and S. Blügel, Phys. Rev. Lett. 86, 4132 (2001).

${ }^{28}$ J. Wiebe, F. Meier, K. Hashimoto, G. Bihlmayer, S. Blügel, P. Ferriani, S. Heinze, and R. Wiesendanger, Phys. Rev. B 72, 193406 (2005).

${ }^{29}$ E. Lundgren, B. Stanka, W. Koprolin, M. Schmid, and P. Varga, Surf. Sci. 423, 357 (1999).

${ }^{30}$ N. Quaas, M. Wenderoth, A. Weismann, R. G. Ulbrich, and K. Schönhammer, Phys. Rev. B 69, 201103 (2004).

${ }^{31}$ J. Tersoff and D. R. Hamann, Phys. Rev. Lett. 50, 1998 (1983).

${ }^{32}$ J. Tersoff and D. R. Hamann, Phys. Rev. B 31, 805 (1985).
${ }^{33}$ A. Weismann, M. Wenderoth, S. Lounis, P. Zahn, N. Quaas, R. G. Ulbrich, P. H. Dederichs, and S. Blügel, Science 323, 1190 (2009).

${ }^{34}$ L. Zhou, F. Meier, J. Wiebe, and R. Wiesendanger, Phys. Rev. B 82, 012409 (2010).

${ }^{35}$ O. Šipr, S. Bornemann, J. Minár, S. Polesya, V. Popescu, A. Šimůnek, and H. Ebert, J. Phys. Condens. Matter 19, 096203 (2007).

${ }^{36}$ A. Mosca Conte, S. Fabris, and S. Baroni, Phys. Rev. B 78, 014416 (2008).

${ }^{37}$ S. Baud, C. Ramseyer, G. Bihlmayer, and S. Blügel, Phys. Rev. B 73, 104427 (2006).

${ }^{38}$ P. Błoński and J. Hafner, J. Phys. Condens. Matter 21, 426001 (2009).

${ }^{39}$ C. Etz, J. Zabloudil, P. Weinberger, and E. Y. Vedmedenko, Phys. Rev. B 77, 184425 (2008).

${ }^{40}$ R. K. Das, R. Misra, S. Tongay, R. Rairigh, and A. F. Hebard, J. Magn. Magn. Mater. 322, 2618 (2010). 